\title{
CONSISTENCY OF REGULARIZATION FOR SCALAR FIELDS
}

\author{
DOUG PICKRELL
}

\begin{abstract}
In two dimensional constructive quantum field theory for scalar fields, it is necessary to regularize both the action and the total (Gaussian) volume. In this paper we consider the compatibility of these regularizations.
\end{abstract}

\section{INTRODUCTION}

Throughout this note, $m_{0}>0$, and $P: \mathbb{R} \rightarrow \mathbb{R}$ is a polynomial which is bounded from below. If $\hat{\Sigma}$ is a closed Riemannian surface, then $\mathcal{D}(\hat{\Sigma})$ and $\mathcal{D}^{\prime}(\hat{\Sigma})$ denote the spaces of smooth and generalized functions (or distributions) on $\hat{\Sigma}$, respectively, and a function $f$ is identified with the distribution $f d A$, where $d A$ is the Riemannian area form. The pairing of a function $f$ and a distribution $\phi$ is denoted by $(f, \phi)$.

The $P(\phi)_{2}$ quantum field theory (in finite volume), corresponding to the parameters $\left(m_{0}, P\right)$, is essentially defined by the Feynman-Kac measure on $\mathcal{D}^{\prime}(\hat{\Sigma})$ given by

$$
\exp \left(-\int_{\hat{\Sigma}}: P\left(\left(\delta_{x}, \phi\right)\right):_{C_{0}} d A(x)\right) \operatorname{det}_{\zeta}\left(\Delta+m_{0}^{2}\right)^{-1 / 2} d \phi_{C},
$$

where $\Delta$ denotes the (nonnegative) Laplacian on $\hat{\Sigma}, \operatorname{det}_{\zeta}$ denotes the zeta function determinant, $C=\left(\Delta+m_{0}^{2}\right)^{-1}, d \phi_{C}$ is the Gaussian measure having Fourier transform

$$
\int_{\mathcal{D}^{\prime}(\hat{\Sigma})} e^{-i(f, \phi)} d \phi_{C}=\exp \left(-\frac{1}{2}(f, C f)\right), \quad f \in \mathcal{D}(\hat{\Sigma}),
$$

$\int_{\hat{\Sigma}}: P\left(\left(\delta_{x}, \phi\right)\right):_{C_{0}}$ denotes point splitting regularization with respect to

$$
C_{0}\left(m_{0} ; x, y\right)=-\frac{1}{2 \pi} \ln \left(m_{0} d(x, y)\right),
$$

and $d(x, y)$ is the distance between the points $x, y \in \hat{\Sigma}$ (see [1], which we use as a general reference, and section 4 of [3]).

It is necessary to regularize $\int P\left(\left(\delta_{x}, \phi\right)\right) d A(x)$, because the measure $d \phi_{C}$ is not supported on ordinary functions. The use of $C_{0}$, rather than $C$, and the inclusion of the zeta function determinant, are essential to show that the measures in (1) lead to a theory which is local in the sense of Segal; see 3 . This leads to the consistency question addressed in the following

Theorem 1.1. Given a closed Riemannian surface $\hat{\Sigma}$, if $m^{2}=m_{1}^{2}+m_{0}^{2}$, then

$$
\begin{aligned}
& \exp \left(-\frac{1}{2} m_{1}^{2} \int_{\hat{\Sigma}}:\left(\delta_{x}, \phi\right)^{2}:_{C_{0}\left(m_{0}, \hat{\Sigma}\right)}\right) \operatorname{det}_{\zeta}\left(\Delta+m_{0}^{2}\right)^{-1 / 2} d \phi_{C\left(m_{0}, \hat{\Sigma}\right)} \\
& \quad=\exp \left(\frac{1}{4 \pi} m_{1}^{2}\left(\ln \left(m_{0} / 4\right)+\gamma\right) A\right) \operatorname{det}_{\zeta}\left(\Delta+m^{2}\right)^{-1 / 2} d \phi_{C(m, \hat{\Sigma})},
\end{aligned}
$$

where $A=\int d A$ and $\gamma$ is Euler's constant. 
Although I do not understand its significance, this Theorem singles out $4 \exp (-\gamma)$ as a special value for the bare mass.

\section{Proof of Theorem 1.1}

Throughout this section $C=C\left(m_{0}, \hat{\Sigma}\right)$ and $C_{0}=C_{0}\left(m_{0}, \hat{\Sigma}\right)$. It is a fundamental fact that

$$
C=C\left(m_{0} ; x, y\right)=C_{0}\left(m_{0} ; x, y\right)+C_{f}\left(m_{0} ; x, y\right),
$$

where $C_{f}$ is a smooth function of $(x, y) \in \hat{\Sigma} \times \hat{\Sigma}$. We refer to $C_{f}$ as the finite part of $C$. Let $\Delta f_{k}=\lambda_{k} f_{k}$, where the $f_{k}$ are normalized eigenfunctions corresponding to the eigenvalues $0=\lambda_{0}<\lambda_{1} \ldots$ We will also write $\int(\cdot)$ for the integral over $\hat{\Sigma}$ with respect to $d A$.

We first recall that

$$
\int:\left(\delta_{x}, \phi\right)^{2}:_{C}=\sum\left(\phi_{k}^{2}-E\left(\phi_{k}^{2}\right)\right)=\sum\left(\phi_{k}^{2}-\frac{1}{m_{0}^{2}+\lambda_{k}}\right),
$$

where $E(\cdot)$ denotes expectation with respect to $d \phi_{C}$. To verify (2), let $\delta_{t, x}=$ $\exp (-t \Delta) \delta_{x}$. Then by definition (see section 6.3 of [1]) the left hand side is the limit as $t \downarrow 0$ of

$$
\begin{gathered}
\int_{x}\left\{\left(\phi, \delta_{t, x}\right)^{2}-\left(\delta_{t, x}, C \delta_{t, x}\right)\right\} \\
=\int_{x}\left\{\int_{y} \int_{z} \delta_{t, x}(y) \delta_{t, x}(z) \phi(y) \phi(z)-\int_{w} \delta_{t, x}(w) C \delta_{t, x}(w)\right\} \\
=\int_{x}\left\{\int_{y} \int_{z} \sum e^{-t\left(\lambda_{j}+\lambda_{k}\right)} f_{j}(x) f_{j}(y) f_{k}(x) f_{k}(z) \phi(y) \phi(z)\right. \\
\left.-\int_{w} \sum e^{-t\left(\lambda_{j}+\lambda_{k}\right)}\left(m_{0}^{2}+\lambda_{k}\right)^{-1} f_{j}(x) f_{j}(w) f_{k}(x) f_{k}(w)\right\} \\
=\int_{x}\left\{\sum e^{-t\left(\lambda_{j}+\lambda_{k}\right)} f_{j}(x) c_{j} f_{k}(x) \phi_{k}\right. \\
\left.-\int_{w} \sum e^{-t\left(\lambda_{j}+\lambda_{k}\right)}\left(m_{0}^{2}+\lambda_{k}\right)^{-1} f_{j}(x) f_{j}(w) f_{k}(x) f_{k}(w)\right\} \\
=\sum\left(e^{-2 t \lambda_{j}} \phi_{j}^{2}-e^{-2 t \lambda_{j}}\left(m_{0}^{2}+\lambda_{j}\right)^{-1}\right) .
\end{gathered}
$$

When we take the limit as $t \rightarrow 0$, we obtain (2). Thus

$$
\int:\left(\delta_{x}, \phi\right)^{2}:_{C_{0}}=\sum\left(\phi_{k}^{2}-\frac{1}{m_{0}^{2}+\lambda_{k}}\right)+\int C_{f}\left(m_{0}, x, x\right) .
$$

We now claim that

$$
\begin{gathered}
\exp \left(-\frac{1}{2} m_{1}^{2} \int:\left(\delta_{x}, \phi\right)^{2}:_{C_{0}}\right) d \phi_{C\left(m_{0}, \hat{\Sigma}\right)} \\
=\exp \left(-\frac{1}{2} m_{1}^{2} \int C_{f}\left(m_{0}, x, x\right)\right) \operatorname{det}_{2}\left(1+m_{1}^{2} C\right)^{-1 / 2} d \phi_{C(m, \hat{\Sigma})},
\end{gathered}
$$

where $\operatorname{det}_{2}$ denotes the Hilbert-Schmidt regularized determinant. This follows from (3) and the following calculation:

$$
\exp \left(-\frac{m_{1}^{2}}{2} \sum\left(\phi_{k}^{2}-\frac{1}{m_{0}^{2}+\lambda_{k}}\right)\right) d \phi_{C}
$$




$$
\begin{gathered}
=\exp \left(-\frac{m_{1}^{2}}{2} \sum\left(\phi_{k}^{2}-\frac{1}{m_{0}^{2}+\lambda_{k}}\right)\right) \prod_{k=0}^{\infty}\left(\frac{m_{0}^{2}+\lambda_{k}}{2 \pi}\right)^{1 / 2} e^{-\frac{m_{0}^{2}+\lambda_{k}}{2} \phi_{k}^{2}} d \phi_{k} \\
=\prod_{k=0}^{\infty} e^{\frac{m_{1}^{2}}{2} \frac{1}{m_{0}^{2}+\lambda_{k}}}\left(\frac{m_{0}^{2}+\lambda_{k}}{m^{2}+\lambda_{k}}\right)^{1 / 2} \prod_{k=0}^{\infty}\left(\frac{m^{2}+\lambda_{k}}{2 \pi}\right)^{1 / 2} e^{-\frac{m^{2}+\lambda_{k}}{2} \phi_{k}^{2}} d \phi_{k} \\
=\prod_{k=0}^{\infty}\left(\left(1+\frac{m_{1}^{2}}{m_{0}^{2}+\lambda_{k}}\right) e^{-\frac{m_{1}^{2}}{m_{0}^{2}+\lambda_{k}}}\right)^{-1 / 2} d \phi_{C(m, \hat{\Sigma})}=\operatorname{det}_{2}\left(1+m_{1}^{2} C\right)^{-1 / 2} d \phi_{C(m, \hat{\Sigma})}
\end{gathered}
$$

Theorem 1.1 is therefore equivalent to the following statement about multiplicative anomalies for zeta function determinants.

\section{Theorem 2.1.}

$\operatorname{det}_{\zeta}\left(m_{1}^{2}+m_{0}^{2}+\Delta\right)=\operatorname{det}_{\zeta}\left(m_{0}^{2}+\Delta\right) \operatorname{det}_{2}\left(1+m_{1}^{2} C\left(m_{0}, \hat{\Sigma}\right)\right) \exp \left(m_{1}^{2} \int\left(C_{f}\left(m_{0} ; x, x\right)-\gamma_{0}\right)\right)$,

where $\gamma_{0}=\frac{1}{2 \pi}\left(\ln \left(\frac{1}{4} m_{0}\right)+\gamma\right)$.

Proof. Let $E=m_{0}^{2}+\Delta=C^{-1}$. Then the left hand side of Theorem 2.1 equals

$$
\operatorname{det}_{\zeta}\left(m_{1}^{2}+E\right)=\operatorname{det}_{\zeta}\left(E\left(1+m_{1}^{2} C\right)\right) \text {. }
$$

As a pseudodifferential operator, $C$ has order -2 . Because $\hat{\Sigma}$ is two dimensional, in general, if $\tilde{C}$ has order -2 , then $\operatorname{tr}\left(E^{-s} \tilde{C}\right)$ is holomorphic for $\operatorname{Re}(s)>0$ and has a Laurent expansion in a neighborhood of $s=0$ of the form

$$
\operatorname{tr}\left(E^{-s} \tilde{C}\right)=\operatorname{Res}(\tilde{C}) \frac{1}{s}+c_{0}+O(s),
$$

where Res is the noncommutative residue and the constant term $c_{0}$ is called the finite part of the trace. In section 3 of [2] we wrote $c_{0}=\mathcal{F} \mathcal{P} \operatorname{tr}\left(E^{-s} \tilde{C}\right)$ (this does possibly depend upon the principal symbol of $E$, so $E$ is included in the notation). By Lemma 3.10 of [2] the above determinant equals

$$
\operatorname{det}_{\zeta}(E) \exp \left(\mathcal{F} \mathcal{P} \operatorname{tr}\left(E^{-s} \log \left(1+m_{1}^{2} C\right)\right)\right) \text {. }
$$

Thus to prove Theorem 2.1] we need to show that

$$
\exp \left(\left(\mathcal{F P} \operatorname{tr}\left(C^{s} \log \left(1+m_{1}^{2} C\right)\right)-m_{1}^{2} \int\left(C_{f}-\gamma_{0}\right)\right)\right)=\operatorname{det}_{2}\left(1+m_{1}^{2} C\right)
$$

or

$$
\mathcal{F P} \operatorname{tr}\left(C^{s} \log \left(1+m_{1}^{2} C\right)\right)-m_{1}^{2} \int\left(C_{f}-\gamma_{0}\right)=\operatorname{tr}\left(\log \left(1+m_{1}^{2} C\right)-m_{1}^{2} C\right)
$$

It suffices to show this for $m_{1}^{2}$ sufficiently small. The right hand side of (5) equals

$$
\operatorname{tr}\left(-\frac{1}{2}\left(m_{1}^{2} C\right)^{2}+\frac{1}{3}\left(m_{1}^{2} C\right)^{3}+\ldots\right),
$$

i.e. we just delete the first term in the expansion of the logarithm.

We now consider the left hand side of (5). For $\operatorname{Re}(s)>0$,

$$
\begin{gathered}
\operatorname{tr}\left(C^{s} \log \left(1+m_{1}^{2} C\right)\right)-m_{1}^{2} \int\left(C_{f}-\gamma_{0}\right) \\
=\operatorname{tr}\left(C^{s} m_{1}^{2} C\right)-m_{1}^{2} \int\left(C_{f}-\gamma_{0}\right)+\operatorname{tr}\left(C^{s}\left(-\frac{1}{2}\left(m_{1}^{2} C\right)^{2}+\frac{1}{3}\left(m_{1}^{2} C\right)^{3}+\ldots\right)\right)
\end{gathered}
$$


The third term extends to an analytic function in a neighborhood of $s=0$, and its value at $s=0$ agrees with the trace in (6). Thus to prove (5) we need to show that for small $s$

$$
\operatorname{tr}\left(C^{s} m_{1}^{2} C\right)-m_{1}^{2} \int\left(C_{f}-\gamma_{0}\right)=\frac{\operatorname{Res}\left(m_{1}^{2} C\right)}{s}+h(s)
$$

where $h(s)$ is holomorphic in a neighborhood of $s=0$ and vanishes at $s=0$. This is implied by the following lemma, which is probably well-known to experts.

\section{Lemma 1.}

$$
\operatorname{tr}\left(C^{s} C\right)=\frac{\operatorname{Res}(C)}{s}+\int\left(C_{f}-\gamma_{0}\right)+O(s),
$$

where $\operatorname{Res}(C)=A / 4 \pi$ and $A=\int d A$.

The left hand side of Lemma 1 equals

$$
\frac{1}{\Gamma(s+1)} \int_{0}^{\infty} t^{s} \operatorname{tr}\left(e^{-t E}\right) d t
$$

We use the asymptotic expansion

$$
\operatorname{tr}\left[e^{-t \Delta}\right]=\frac{1}{4 \pi t} \int d A+\frac{1}{12 \pi} \int r_{g} d A+O(t), \quad \text { as } \quad t \downarrow 0,
$$

where $r_{g}$ denotes scalar curvature. This expansion implies

$$
\begin{gathered}
\operatorname{tr}\left[e^{-t E}\right]=\left(e^{-t m_{0}^{2}}\right)\left(\frac{1}{4 \pi t} A+\frac{1}{12 \pi} \int_{\Sigma} r_{g} d A+O(t)\right) \\
=\frac{1}{4 \pi t} A+\frac{1}{12 \pi} \int_{\Sigma} r_{g} d A-\frac{m_{0}^{2}}{4 \pi} A+O(t)
\end{gathered}
$$

as $t \downarrow 0$. Then (7) equals

$$
\begin{gathered}
\frac{1}{\Gamma(s+1)} \int_{0}^{\infty} t^{s} \frac{A}{4 \pi t} d t+\frac{1}{\Gamma(s+1)} \int_{0}^{\infty} t^{s}\left\{\operatorname{tr}\left(e^{-t E}\right)-\frac{A}{4 \pi t}\right\} d t \\
=\frac{A}{4 \pi} \frac{1}{s}+\frac{1}{\Gamma(s+1)} \int_{0}^{\infty} t^{s}\left\{\operatorname{tr}\left(e^{-t E}\right)-\frac{A}{4 \pi t}\right\} d t
\end{gathered}
$$

The second term is holomorphic in a neighborhood of $s=0$. Thus

$$
\operatorname{tr}\left(C^{s+1}\right)=\frac{A}{4 \pi} \frac{1}{s}+\int_{0}^{\infty}\left\{\operatorname{tr}\left(e^{-t E}\right)-\frac{A}{4 \pi t}\right\} d t+O(s)
$$

This implies that $\operatorname{Res}(C)=A / 4 \pi$.

Remark. The residue can be calculated in a second way. If $\alpha$ denotes the canonical one-form on $T^{*} \hat{\Sigma}$, and $\omega=d \alpha$, then because the principal symbol of $C$ is $|p|^{-2}$ (as a function on $\left.T^{*} \hat{\Sigma}\right)$,

$$
\operatorname{Res}(C)=\frac{1}{(2 \pi)^{2}} \int_{S\left(T^{*} \Sigma\right)}|p|^{-2} \alpha d \alpha=\frac{1}{(2 \pi)^{2}} \int_{|p|^{2} \leq 1} \omega \wedge \omega .
$$

In local coordinates, if $p=\sum p_{j} d q^{j}$, then $|p|^{2}=g^{i, j} p_{i} p_{j}$, and the integral in (9) has the local expression

$$
\frac{1}{(2 \pi)^{2}} \int_{q}\left(\int_{|p|^{2} \leq 1} d p_{1} d p_{2}\right) d q^{1} d q^{2}=\frac{1}{(2 \pi)^{2}} \int_{q} \pi\left(\operatorname{det}\left(g_{i, j}\right)^{1 / 2}\right) d q^{1} d q^{2}=\frac{1}{4 \pi} \int d A .
$$


Given (8), to complete the proof of the Lemma, we need to show

$$
\int_{0}^{\infty}\left\{\operatorname{tr}\left(e^{-t E}\right)-\frac{A}{4 \pi t}\right\} d t=\int\left(C_{f}(x, x)-\gamma_{0}\right) .
$$

The left hand side equals

$$
\begin{gathered}
\lim _{T \downarrow 0} \int_{T}^{1 / T}\left\{-\frac{d}{d t} \operatorname{tr}\left(e^{-t E} C\right)-\frac{A}{4 \pi t}\right\} d t \\
=\lim _{T \downarrow 0}\left(\operatorname{tr}\left(e^{-T E} C-e^{-T^{-1} E} C\right)+\frac{A}{2 \pi} \ln (T)\right) \\
=\operatorname{tr}\left(C_{f}\right)+\lim _{T \downarrow 0}\left(\operatorname{tr}\left(e^{-T E} C_{0}\right)+\frac{A}{2 \pi} \ln (T)\right) \\
=\operatorname{tr}\left(C_{f}\right)+\lim _{T \downarrow 0}\left(\operatorname{tr}\left(e^{-T E}\left(-\frac{1}{2 \pi} \ln (d(x, y))\right)+\frac{A}{2 \pi} \ln (T)\right)-\frac{A}{4 \pi} \ln \left(m_{0}^{2}\right)\right) .
\end{gathered}
$$

We now calculate

$$
\begin{gathered}
\lim _{T \downarrow 0}\left(\operatorname{tr}\left(e^{-T E} \ln (d(x, y))-A \ln T\right)\right. \\
=\lim _{T \downarrow 0}\left(\int_{y} \int_{x} e^{-T E}(y, x) \ln (d(x, y))-A \ln (T)\right) .
\end{gathered}
$$

For small $T$ the double integral is concentrated near the diagonal. For fixed $y$, there is an asymptotic expansion

$$
e^{-T E}(y, x)=\frac{1}{4 \pi T} \exp (-d(x, y) / 4 T)(1+O(T))
$$

In exponential coordinates centered at $y, d A=j(v) d \lambda(v)$, where $j(v)=1+O\left(r^{2}\right)$, $r=|v|$, and $d \lambda(v)$ denotes the Riemannian volume for $v \in T_{y}$. Thus (12) equals

$$
\begin{gathered}
\lim _{T \downarrow 0}\left(\int_{y} \int_{x} \frac{1}{4 \pi T} e^{-d(x, y)^{2} / 4 T}(1+O(T)) \ln (d(x, y))-A \ln (T)\right) \\
=\lim _{T \downarrow 0}\left(\int_{y} \int_{x} \frac{1}{4 \pi T} e^{-d(x, y)^{2} / 4 T} \ln (d(x, y))-A \ln (T)\right) \\
=\lim _{T \downarrow 0}\left(\int_{y}\left(\int_{v \in T_{y}} \frac{1}{4 \pi T} e^{-r^{2} / 4 T} \ln (r) j(v) d \lambda(v)-\ln (T)\right)\right) \\
=A \lim _{T \downarrow 0}\left(\int_{0}^{\infty} e^{-r^{2} / 4 T}\left(\ln \left(r^{2} / 4 T\right)+\ln (4 T) d\left(r^{2} / 4 T\right)-\ln (T)\right),\right. \\
\text { because } \int \frac{1}{4 \pi T} e^{-r^{2} / 4 T} \ln (r) O\left(r^{2}\right) d \lambda(v) \rightarrow 0 \text { as } T \rightarrow 0 \\
=A\left(\ln (4)+\left(\int_{0}^{\infty} e^{-u}(\ln (u) d u)=A(\ln (4)-\gamma) .\right.\right.
\end{gathered}
$$

When we plug this into (11), we obtain (10). This completes the proof of Lemma 1 and Theorem 1.1 . 


\section{Conformally Invariant Background}

As in the previous section $\hat{\Sigma}$ is a Riemannian surface. As in Section 4 of [3], $d \phi_{C(0, \hat{\Sigma})}$ denotes the infinite conformally invariant measure on generalized functions $\phi=\sum \phi_{n} f_{n}$ given by

$$
d \phi_{C(0, \hat{\Sigma})}=d \lambda\left(\phi_{0}\right) \prod_{k=1}^{\infty}\left(\frac{\lambda_{k}}{2 \pi}\right)^{1 / 2} e^{-\frac{\lambda_{k}}{2} \phi_{k}^{2}} d \phi_{k}
$$

\section{Lemma 2.}

$$
\lim _{m_{0} \downarrow 0} \operatorname{det}_{\zeta}\left(m_{0}^{2}+\Delta\right)^{-1 / 2} d \phi_{C\left(m_{0}, \hat{\Sigma}\right)}=\frac{1}{\sqrt{2 \pi}} \operatorname{det}_{\zeta}^{\prime}(\Delta)^{-1 / 2} d \phi_{C(0, \hat{\Sigma})}
$$

Proof. On the one hand

$$
\begin{gathered}
\operatorname{det}_{\zeta}\left(m_{0}^{2}+\Delta\right)^{-1 / 2}=\exp \left(\left.\frac{1}{2} \frac{d}{d s}\right|_{s=0} \sum_{n=0}^{\infty}\left(m_{0}^{2}+\lambda_{n}\right)^{-s}\right) \\
=m_{0}^{-1} \exp \left(\left.\frac{1}{2} \frac{d}{d s}\right|_{s=0} \sum_{n=1}^{\infty}\left(m_{0}^{2}+\lambda_{n}\right)^{-s}\right)
\end{gathered}
$$

On the other hand

$$
\begin{gathered}
d \phi_{C\left(m_{0}, \hat{\Sigma}\right)}=\prod_{k=0}^{\infty}\left(\frac{m_{0}^{2}+\lambda_{k}}{2 \pi}\right)^{1 / 2} e^{-\frac{m_{0}^{2}+\lambda_{k}}{2} \phi_{k}^{2}} d \phi_{k} \\
\frac{m_{0}}{\sqrt{2 \pi}} \exp \left(-\frac{m_{0}^{2}}{2} \phi_{0}^{2}\right) d \lambda\left(\phi_{0}\right) \prod_{k=1}^{\infty}\left(\frac{m_{0}^{2}+\lambda_{k}}{2 \pi}\right)^{1 / 2} e^{-\frac{m_{0}^{2}+\lambda_{k}}{2} \phi_{k}^{2}} d \phi_{k}
\end{gathered}
$$

When we form the product, there is a cancellation involving $m_{0}$,

$$
\begin{gathered}
\operatorname{det}_{\zeta}\left(m_{0}^{2}+\Delta\right)^{-1 / 2} d \phi_{C\left(m_{0}, \hat{\Sigma}\right)}= \\
\frac{1}{\sqrt{2 \pi}} \exp \left(\left.\frac{1}{2} \frac{d}{d s}\right|_{s=0} \sum_{n=1}^{\infty}\left(m_{0}^{2}+\lambda_{n}\right)^{-s}\right) \exp \left(-\frac{m_{0}^{2}}{2} \phi_{0}^{2}\right) d \lambda\left(\phi_{0}\right) \\
\prod_{k=1}^{\infty}\left(\frac{m_{0}^{2}+\lambda_{k}}{2 \pi}\right)^{1 / 2} e^{-\frac{m_{0}^{2}+\lambda_{k}}{2} \phi_{k}^{2}} d \phi_{k}
\end{gathered}
$$

When we take the limit as $m_{0} \rightarrow 0$, we obtain the right hand side of Lemma 2 completing the proof.

Recall that the measures $d \phi_{C(m, \hat{\Sigma})}$ are mutually absolutely continuous, for $0 \leq$ $m<\infty$; see Lemma 3 of 3 . Suppose that $\sigma>0$. Then

$$
\begin{gathered}
e^{-\frac{1}{2} \sigma \int_{\hat{\Sigma}}:\left(\delta_{x}, \phi\right)^{2}:_{C_{0}(m, \hat{\Sigma})}} \operatorname{det}_{\zeta}\left(m_{0}^{2}+\Delta\right)^{-1 / 2} d \phi_{C\left(m_{0}, \hat{\Sigma}\right)} \\
=e^{-\frac{1}{2} \sigma \int_{\hat{\Sigma}}:\left(\delta_{x}, \phi\right)^{2}:_{C_{0}(m, \hat{\Sigma})}} e^{\frac{1}{2} \sigma \int_{\hat{\Sigma}}:\left(\delta_{x}, \phi\right)^{2}:_{C_{0}\left(m_{0}, \hat{\Sigma}\right)}} \\
e^{-\frac{1}{2} \sigma \int_{\hat{\Sigma}}:\left(\delta_{x}, \phi\right)^{2}:_{C_{0}\left(m_{0}, \hat{\Sigma}\right)}} \operatorname{det}_{\zeta}\left(m_{0}^{2}+\Delta\right)^{-1 / 2} d \phi_{C\left(m_{0}, \hat{\Sigma}\right)} \\
=\left(\frac{m}{m_{0}}\right)^{\frac{\sigma A}{4 \pi}} \exp \left(\sigma \gamma_{0} A\right) \operatorname{det}_{\zeta}\left(\sigma+m_{0}^{2}+\Delta\right)^{-1 / 2} d \phi_{C\left(\sqrt{\sigma+m_{0}^{2}}, \hat{\Sigma}\right)} \\
=(m \exp (\gamma-\ln (4)))^{\frac{\sigma A}{4 \pi}} \operatorname{det}_{\zeta}\left(\sigma+m_{0}^{2}+\Delta\right)^{-1 / 2} d \phi_{C\left(\sqrt{\sigma+m_{0}^{2}}, \hat{\Sigma}\right)}
\end{gathered}
$$

By taking the limit as $m_{0} \rightarrow 0$, using Lemma 2, we obtain 


\section{Theorem 3.1.}

$$
\begin{aligned}
& e^{-\frac{1}{2} \sigma \int_{\hat{\Sigma}}:\left(\delta_{x}, \phi\right)^{2}:_{C_{0}(m, \hat{\Sigma})} \operatorname{det}_{\zeta}^{\prime}(\Delta)^{-1 / 2} d \phi_{C(0, \hat{\Sigma})}} \\
& =\left(\frac{m}{4} \exp (\gamma)\right)^{\frac{\sigma A}{4 \pi}} \operatorname{det}_{\zeta}(\sigma+\Delta)^{-1 / 2} d \phi_{C(\sqrt{\sigma}, \hat{\Sigma})}
\end{aligned}
$$

In Segal's approach to qft, the map $\Sigma \rightarrow \operatorname{Area}(\Sigma)$ defines an additive homomorphism from the category of Riemannian surfaces to $\mathbb{R}$. This is the significance of the term involving area.

\section{REFERENCES}

[1] J. Glimm and A. Jaffe, Quantum Physics, a Functional Integral Point of View, SpringerVerlag (1981).

[2] D. Pickrell, On the action of the group of diffeomorphisms of a surface on sections of the determinant line bundle, Pac. J. Math., Vol. 193, No. 1 (2000) 177-199.

[3],$- P(\phi)_{2}$ quantum field theories and Segal's axioms, Commun. Math. Phys. 280 (2008) 403-425.

E-mail address: pickrell@math.arizona.edu 